Eur. J. Clin. Chem. Clin. Biochem.

Vol. 30, 1992, pp. 357-361

(C) 1992 Walter de Gruyter \& Co. Berlin · New York

\title{
Performance Characteristics of Creatine Kinase-MB Isoenzyme Measured with an Immunoenzymometric and an Immunoinhibition Assay in Acute Myocardial Infarction with and without Thrombolytic Therapy
}

\author{
By Vibeke Schiøler ${ }^{1}, J$. Thode ${ }^{1}$ and E. Kjøller ${ }^{2}$ \\ 1 Department of Clinical Chemistry \\ ${ }^{2}$ Department of Cardiology, The Myocardioscintigraphy in Acute Ischaemic Syndrome study-group*) \\ Herlev University Hospital, Denmark
}

(Received January 2/April 6, 1992)

\begin{abstract}
Summary: In a time study we compared the analytical and clinical performance of the Tandem Icon QSR CK-MB enzyme-immunoassay (Hybritech) (creatine kinase-MB) and a creatine kinase-MB immunoinhibition method (Boehringer Mannheim $\mathrm{GmbH}$ ) (creatine kinase-B). Two hundred and ninety-nine serum samples from 38 patients suspected of acute myocardial infarction were collected at regular intervals during 48 hours. Twenty-nine patients were diagnosed as having acute myocardial infarction, of whom 19 recieved thrombolytic therapy. Although highly correlated, the large scatter around the regression line at low values indicated a different clinical performance of the two methods. We evaluated and compared test performance at different decision levels by means of frequency distributions and predictive values of positive and negative results. For early diagnosis of acute myocardial infarction (4 hours after onset of pain) the Hybritech creatine kinase-MB method gives acceptable predictive values. In thrombolytic treated acute myocardial infarction patients, the peak creatine kinase-MB and creatine kinase-B concentrations were reached after $13.0 \mathrm{~h}$ and $13.6 \mathrm{~h}$ after the onset of pain, compared with $19.8 \mathrm{~h}$ and $17.8 \mathrm{~h}$ for patients without thrombolytic therapy.
\end{abstract}

\section{Introduction}

Thrombolytic therapy in patients with acute myocardial infarction is now standard treatment $(1,2)$ and is most effective when started as soon as possible after onset of symptoms. Therefore an early diagnostic test in patients with suspected acute myocardial infarction is important. The standard laboratory procedures $(3-5)$ are measurement of enzymes, especially creatine kinase isoenzyme $\left.{ }^{1}\right) \mathrm{MB}$, released from the necrotic myocardium.

\footnotetext{
*) Study-group members:

Erik Kjøller, Finn Nielsen, Jørn Carlsen, Knud Skagen, Steen Levin Nielsen, Inge-Lis Kanstrup, Keld Hvid-Jacobsen, Jacob Trier Møller, Per Føge Jensen.

1) Enzyme: Creatine kinase (EC 2.7.3.2) isoenzyme MB.
}

The three most common groups of methods for measurement of creatine kinase isoenzymes are: separation methods (electrophoresis, ion-exchange chromatography) and immunoprecipitation and immunoinhibition methods, which all measure catalytic activity, and specific antibody immunoassays which measure mass concentration $(6-9)$.

In a time study we evaluated the Tandem Icon QSR CK-MB assay (Hybritech) (creatine kinase-MB) and the creatine kinase-MB immunoinhibition method (Boehringer) (creatine kinase-B) in 299 samples from 38 patients suspected of acute myocardial infarction, with respect to predictive values of positive and negative results at different decision limits (frequency distribution). Using both methods, we investigated the influence of thrombolytic therapy on the time 
taken to attain peak values (time to peak) in 29 acute myocardial infarction patients, of whom 19 had received thrombolytic therapy.

\section{Materials and Methods}

\section{Analytical methods}

Creatine kinase-MB mass concentration was assayed with the Tandem Icon QSR CK-MB quantitative test (Hybritech Inc. San Diego, California) as specified by the manufacturer. This assay is specific for creatine kinase-MB isoenzyme and uses two monoclonal antibodies, one binding to the M-subunit and the other to the B-subunit of the creatine kinase-MB molecule. Each test cylinder contains test zones for low and high calibrator and for duplicate sample. Results were measured on a reflectometer (Hybritech Icon Reader). The detection limit was $2 \mu \mathrm{g} / \mathrm{l}$ (manufacturer's specifications).

Creatine kinase-MB catalytic activity (creatine kinase-B) was assayed with creatine kinase-MB (N-acetyl cysteine (NAC)activated) reagents (Boehringer Mannheim GmbH Diagnostica, Mannheim, Germany) at $37^{\circ} \mathrm{C}$ (our routine laboratory method). After selective inhibition of the creatine kinase-M subunit, the residual creatine kinase-B activity was measured. Results were corrected for adenylate kinase activity. This assay also measures creatine kinase-BB and macro creatine kinase (7) when present. The method was adapted to the RA 1000 autoanalyser (Technicon Instruments Corporation, Tarrytown, USA).

\section{Patients}

We assayed 299 blood samples from 38 patients. Blood was collected from the antecubital vein at approximately $0,3,6,9$, $12,18,24,36$ and 48 hours after admission. For some patients the sample series were not complete. The samples were centrifuged and serum was stored at $4-8{ }^{\circ} \mathrm{C}$ for measurement of creatine kinase-B within 24 hours in our in-house routine assay, and at $-70^{\circ} \mathrm{C}$ for measurement of creatine kinase-MB within 2 months.

All samples assayed were from patients admitted to the coronary care unit with acute ischaemic syndrome and included in the Myocardioscintigraphy in Acute Ischaemic Syndrome project. The diagnosis of acute myocardial infarction was established on the basis of clinical history, daily 12-lead electrocardiogram and enzyme results, not including creatine kinase-B and creatine kinase-MB. Twenty-nine patients were diagnosed as having acute myocardial infarction, and 19 of these recieved thrombolytic therapy (streptokinase $1.5 \times 10^{6} \mathrm{IU}$ ).

The patients were admitted $3 \frac{1}{2}$ hours (mean) (range $2 \frac{1}{2}$ to 14 hours) after the onset of pain. In 26 of the patients the first blood sample was collected within 4 hours after onset of pain and in 9 patients the first sample was collected so close to 4 hours that extrapolation from the time curve could be applied.

\section{Statistical method}

Demings correlation test was used for comparison of creatine kinase- $\mathrm{B}$ and creatine kinase-MB results (10)

\section{Results}

\section{Analytical imprecision}

Creatine kinase-MB between-assay coefficient of variation $(\mathrm{CV})$ for a high (mean $21 \mu \mathrm{g} / \mathrm{l} ; \mathrm{N}=10)$ and a low (mean $5 \mu \mathrm{g} / \mathrm{l}) ; \mathrm{N}=10$ ) patient serum pool were $6 \%$ and $15 \%$ respectively. Creatine kinase-B betweenassay $\mathrm{CV}$ was $5 \%$ (mean $40 \mathrm{U} / 1, \mathrm{~N}=12$ ) and $25 \%$ (mean $10 \mathrm{U} / \mathrm{l}, \mathrm{N}=38$ ).

\section{Analytical correlation}

The correlation between creatine kinase-MB mass concentration (y) and creatine kinase-MB enzyme catalytic activity $(\mathrm{x})$ is shown in figure 1 . The regression equation was: $y=2.078 x-13.826, r=0.946 ; S_{\mathrm{y}, \mathrm{x}}$ $=15.014$. The mean specific enzyme activity $(\overline{\mathrm{x}} / \overline{\mathrm{y}})$ was $560 \mathrm{U} / \mathrm{mg}$. A considerable scatter of the data is observed in the low range (fig. 1, insert), due to the high $\mathrm{CV}$ of the creatine kinase- $\mathrm{B}$ method at low values. For values $<50 \mu \mathrm{g} / \mathrm{l}(\mathrm{U} / \mathrm{l})$ the regression equation was: $y=0.985 x+1.713, r=0.611 ; S_{\mathrm{y}, \mathrm{x}}$ $=11.113$.

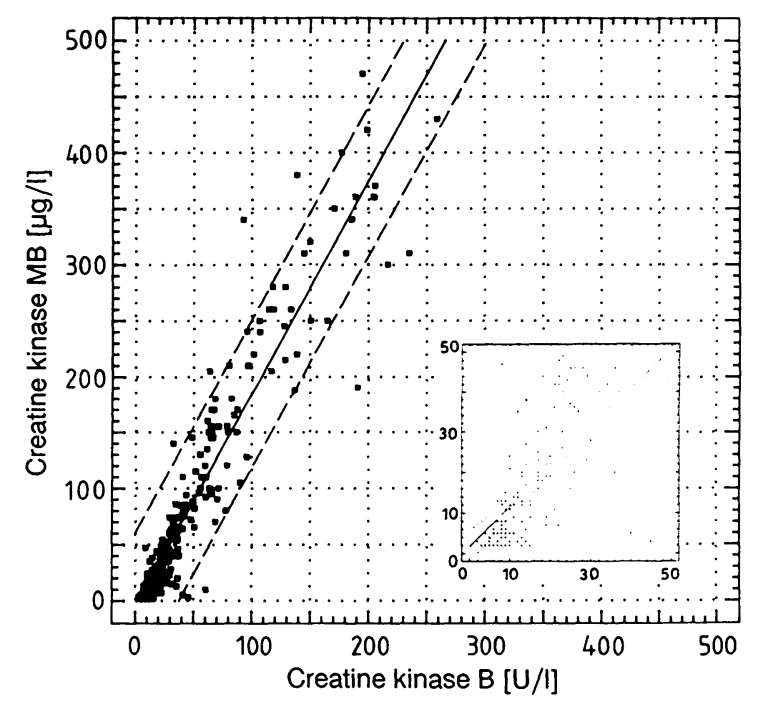

Fig. 1. Correlation between creatine kinase-MB enzyme activity (creatine kinase-B) and creatine kinase-MB mass concentration (creatine kinase-MB) in serum samples from 38 patients suspected of acute myocardial infarction.

56 results were below the detection limit of the creatine kinase-MB method $(2 \mu \mathrm{g} / \mathrm{l})$ and were not included in the calculation. Solid line: $100 \%$ correlation line; dotted lines: standard error of the estimate $\left( \pm S_{y, x}\right)$. Insert shows values $<50 \mu \mathrm{g} / \mathrm{l}(\mathrm{U} / \mathrm{l})$.

\section{Frequency distribution}

Histograms (11) for test results were drawn for both tests (fig. 2a and 2b). At 4 and 8 hours after onset of pain and at peak enzyme concentration, the predictive values of positive (PVpos) and negative (PVneg) test results were calculated at decision limits $2,6,12,20$ and $30 \mu \mathrm{g} / 1$ for creatine kinase-MB and 2, 6, 9, 12, $20,30,50$ and $60 \mathrm{U} / 1$ for creatine kinase-B. The 8- 


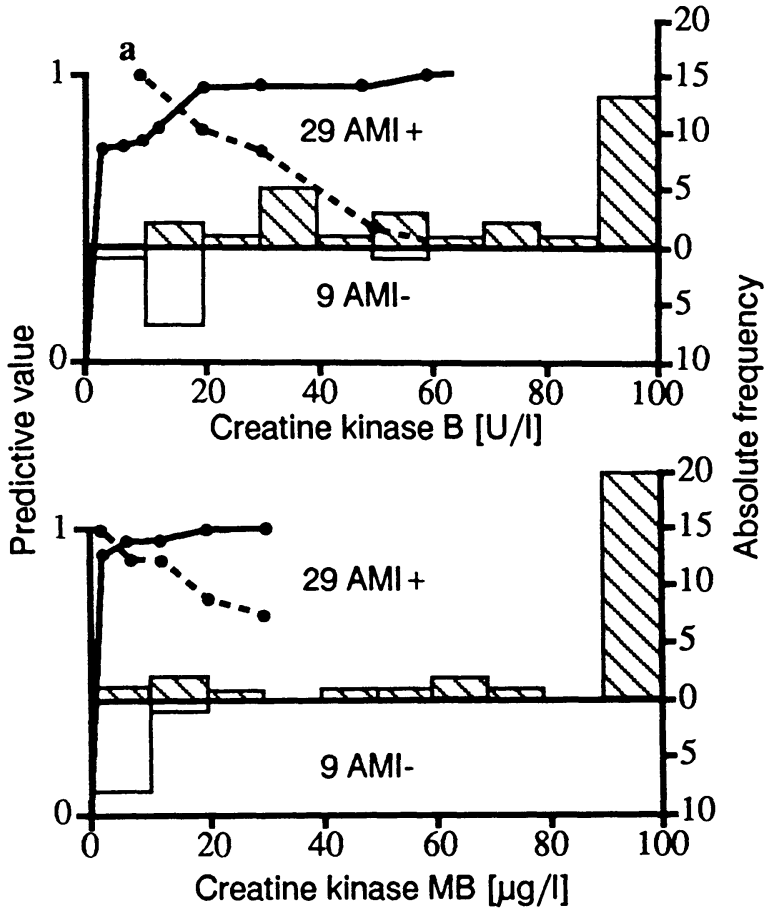

hour values were similar to the peak values and therefore not shown.

As observed both a peak values and at four hours, the creatine kinase-MB test showed highest PVpos at all decision limits. At four hours, PVpos $>0.9$ and a PVneg $\geq 0.75$ were obtained at the decision limit of $2 \mu \mathrm{g} / 1$ for creatine kinase-MB. A similar PVpos was obtained for creatine kinase-B at the decision limit of $12 \mathrm{U} / 1$, however with PVneg $<0.5$.

Time course of creatine kinase-MB and creatine kinase-B

After admission to hospital, 38 patients were followed with blood sampling during a 48-hour period (range $20-84$ hours). For 29 patients with acute myocardial infarction, 19 receiving (a) and 10 not receiving thrombolytic therapy (b), curves were drawn for crea-

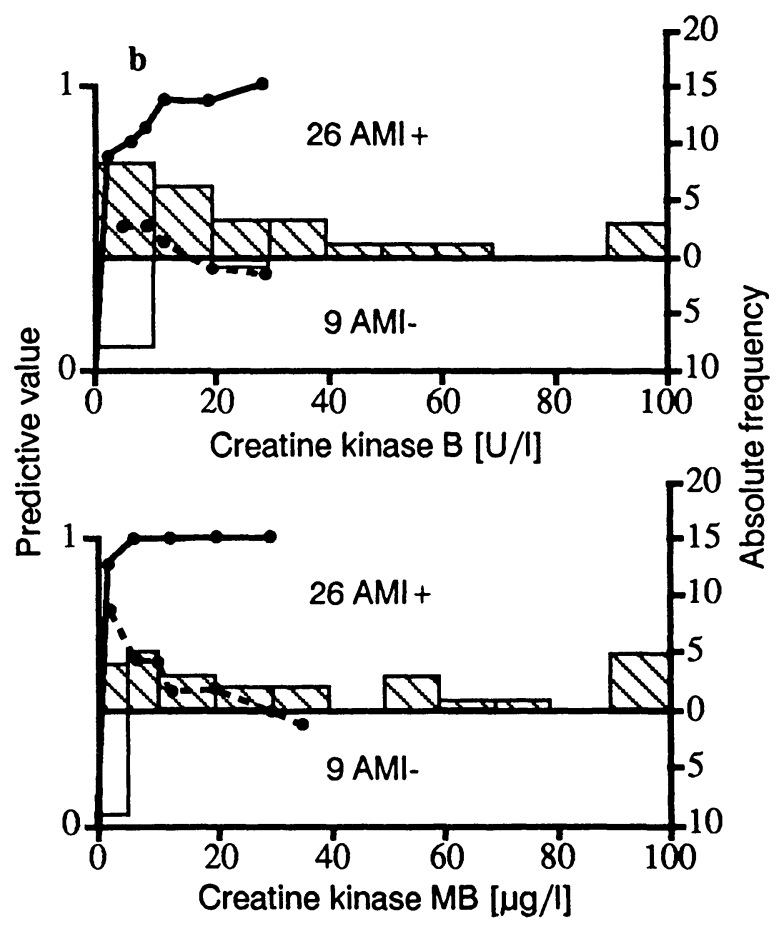

Fig. 2a, b. Frequency distribution based on creatine kinase-B (upper field) and creatine kinase-MB (lower field) peak values (a) for 29 acute myocardial infarctions (AMI; hatched bars) and 9 non-acute myocardial infarctions (non-AMI; open bars) cases and 4-hour values (b) for 26 acute myocardial infarction (hatched bars) and 9 non-acute myocardial infarction (open bars) cases. Right ordinate: number of cases.

All values $>90 \mathrm{U} / \mathrm{l}(\mu \mathrm{g} / \mathrm{l})$ are included in the $90-$ 100 bar.

PVpos (- $)$ and PVneg (-------) curves are plotted (scale left ordinate).
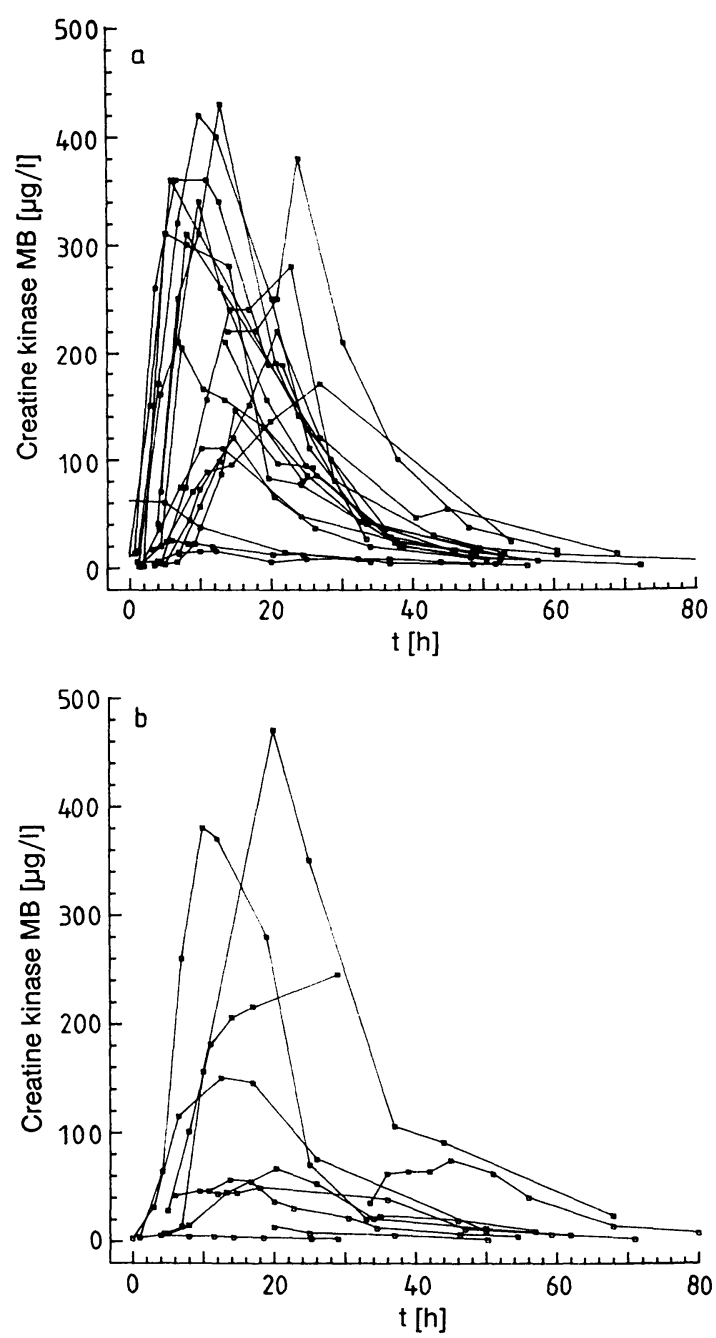

Fig. 3. Time-course of creatine kinase-MB mass concentration in serum samples from 19 acute myocardial infarction patients treated with thrombolytic therapy (a) and in 10 acute myocardial infarction patients without thrombolytic therapy (b). 

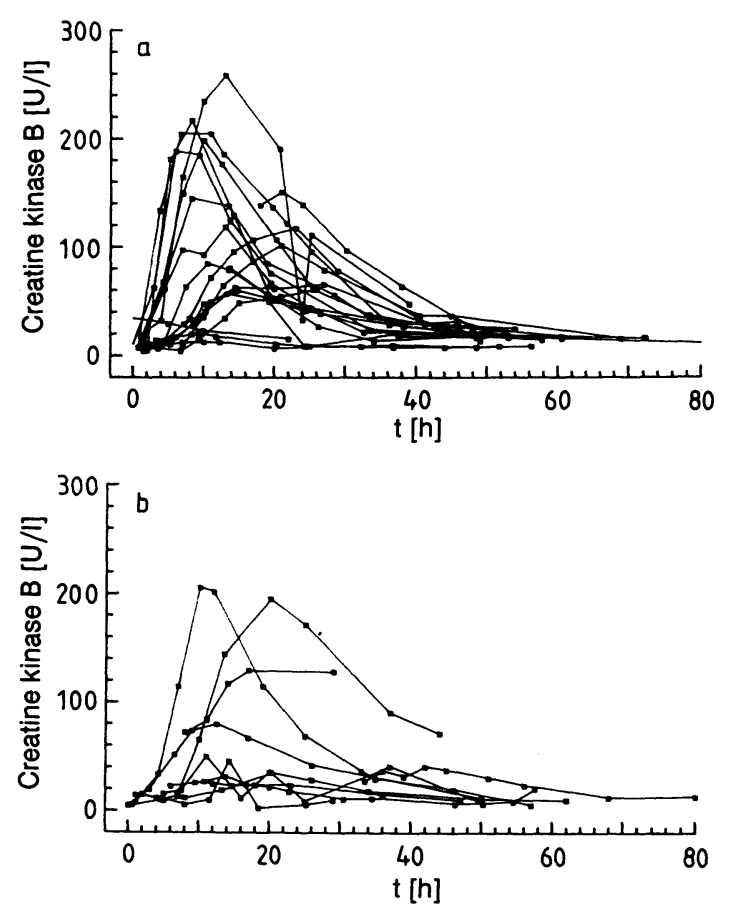

Fig. 4. Time-course of creatine kinase-B enzyme activity in serum samples from 19 acute myocardial infarction patients treated with thrombolytic therapy (a) and in 10 acute myocardial infarction patients without thrombolytic therapy (b).

tine kinase-MB (fig. 3a and 3b) and creatine kinase-B (fig. $4 \mathrm{a}$ and $4 \mathrm{~b}$ ), using the onset of pain as time zero.

Using the decision limit $9 \mu \mathrm{g} / \mathrm{l}$ for creatine kinase-MB and $20 \mathrm{U} / 1$ for creatine kinase-B we found that in the patients receiving thrombolytic therapy, the duration of positive creatine kinase-MB was $48.3 \mathrm{~h}(16-72)$ compared with $43.0 \mathrm{~h}(23-65)$ for creatine kinase-B (NS). Time to peak was $13.0 \mathrm{~h}(2.5-26.0)$ for creatine kinase-MB, compared with $13.6 \mathrm{~h}(3.0-26.0)$ for creatine kinase-B (NS). In the patients who did not receive thrombolytic therapy, the duration of positive test results was $51.6 \mathrm{~h}(42-68)$ for creatine kinase$\mathrm{MB}$ and $31.5 \mathrm{~h}(17-46)$ for creatine kinase-B $(\mathrm{p}<0.01)$. Time to peak was $19.8 \mathrm{~h}(10-45)$ for creatine kinase-MB, compared with $17.8 \mathrm{~h}(10-42)$ for creatine kinase-B (NS).

Although both methods gave different mean values for the time to peak in patients with and without thrombolytic treatment, this was not statistically significant, and probably the result of using a small number of patients.

\section{Discussion}

Our objectives were to investigate the conceivable advantage in using one of the new specific tests for creatine kinase-MB, especially in the early diagnosis of acute myocardial infarction, which may support the use of thrombolytic therapy of acute myocardial infarction (7).

As observed, the creatine kinase-MB mass concentration and the creatine kinase-MB enzyme activity correlated well for elevated enzyme concentrations, in agreement with other studies $(12,13)$. We did not multiply our enzyme activity results by a factor 2 (to compensate for the blocking of the $\mathrm{M}$-subunit), which explains the lower specific activity in our study compared with others. However, at low values $(<50 \mathrm{U} / \mathrm{l})$ the correlation was poor (note the large scatter at low values in figure 1 (insert)), due to the high imprecision of both tests in this range, especially the creatine kinase-B.

The frequency distribution histograms showed that the creatine kinase-MB mass concentration assay could be used in early diagnosis (4 hours after onset of pain) with a PVpos greater than 0.9, combined with a PVneg greater than 0.75 , which was never obtained for the creatine kinase-MB activity method. At the enzyme peak concentration, comparable predictive values were obtained for both methods. At the peak concentration, acceptable clinical performance was obtained for the creatine kinase-MB activity method, using the decision limits of 12 and $20 \mathrm{U} / 1$. At 8 hours after the onset of pain the performance characteristics for both methods were very similar to those at enzyme peak concentrations (not shown). Based on the decision limits of $9 \mu \mathrm{g} / \mathrm{l}$ for creatine kinase-MB and $20 \mathrm{U} / 1$ for creatine kinase- $\mathrm{B}$, the duration time for the positive test in patients who not receiving thrombolytic therapy was significantly longer for creatine kinase-MB than for creatine kinase-B. In patients receiving thrombolytic therapy, the duration time for both methods was not significantly different.

In agreement with other studies $(14,15)$, both methods showed a difference in time to peak between thrombolytic treated patients and non-treated patients; but in our study this difference was not significant $(\mathrm{p}<0.1$ for creatine kinase-MB and $\mathrm{p}<0.25$ for creatine kinase-B), probably due to the small number of patients.

We conclude that both methods provide sufficient predictive values for both positive and negative diagnosis of acute myocardial infarction at eight hours after the onset of pain. The creatine kinase-MB also gives acceptable predictive values for early diagnosis (4 hours after onset of pain), which is claimed to be crucial for successful thrombolytic therapy (1) and may therefore be of value to the clinician as a diagnostic support in the treatment of acute myocardial 
infarction. The appropriate decision limits should be chosen from the frequency distribution histograms, with due consideration for the prevalence and the costs of false results.

\section{References}

1. Koren, G., Luria, M. H., Weiss, A. T., Kriwisky, M. Mosseri, M., Lotan, C., Appelbaum, D., Welber, S., Sapoznikov, D., Ben-David, Y., Hasin, Y. \& Gotsman, M. S. (1987) Early treatment of acute myocardial infarction with intravenous streptokinase. Arch. Intern. Med. 147, 237-240.

2. Simmoons, L. M., Serruys, P. W., van den Brand, M., Res, J., Verheugt, F. W. A., Krauss, X. H., Remme, W. J., Bär, F., de Zwaan, C., van der Laarse, A., Vermeer, F. \& Lubsen, J. (1986) Early thrombolysis in acute myocardial infarction: Limitation of infarct size and improved survival. J. Am. Coll. Cardiol. 7, 717-728.

3. Panteghini, M. (1988) Serum insoforms of creatine kinase isoenzymes. Clin. Biochem. 21, 211-218.

4. Lee, T. H., Weisberg, M. C., Cook, E. F., Daley, K., Brand, D. A. \& Goldman, L. (1987) Evaluation of creatine kinase$\mathrm{MB}$ for diagnosing myocardial infarction. Arch. Intern. Med. 147, 115-121.

5. Hørder, M., Jørgensen, R. J., Hafkenscheid, J. C. M., Carstensen, C. A., Bachmann, C., Bauer, K., Neuwald, C., Rosalki, S. B. \& Ying Foo, A. (1991) Creatine kinase determination: A european evaluation of the creatine kinase determination in serum, plasma and whole blood with the Reflotron system. Eur. J. Clin. Chem. Clin. Biochem. 29, $691-696$.

6. Schwatz, J. G., Brown, R. W., McMahan, A., Gage, C. L. \& Herber, S. A. (1989) Clinical and analytical evaluation of different methods for measurement of creatine kinase isoenzyme MB. Clin. Chem. 35, 130-134.

7. Moerman, J., Nellens, P., Beckers, F. \& Sierens, L. (1986) Diagnostic value of an immunoenzymetric assay compared with an enzymic immunoinhibition procedure for creatine kinase isoenzyme MB. Clin. Chim. Acta 160, 313-317.

\section{Acknowledgements}

We wish to thank Hybritech Inc. Belgium for donation of Tandem Icon reagents.

8. Wu, A. H. B., Gornet, T. G., Harker, C. C. \& Chen, H. (1989) Role of rapid immunoassays for urgent ("stat") determinations of creatine kinase isoenzyme MB. Clin. Chem. 35, 1752-1756.

9. Jørgensen, P., Hørder, M., Selmer, J. \& Bøtker, H. E. (1990) Analytical evaluation of a sensitive enzyme immunoassay for determination of creatine kinase isoenzyme MB. Clin. Chem. 36, 1502-1505.

10. Deming, W. E. (1943) Statistical adjustment of data, John Wiley and Sons. New York NY, p. 184.

11. Gerhardt, W. \& Keller, H. (1986) Evaluation of test data from clinical studies. Scand. J. Clin. Lab. Invest. 46, suppl. 181.

12. Delanghe, J. R., De Mol, A. M., De Buyzere, M. L., De Scheerder, I. K. \& Wieme, R. J. (1990) Mass concentration and activity concentration of creatine kinase isoenzyme MB compared in serum after acute myocardial infarction. Clin. Chem. 36, 149-153.

13. Murthy, V. V. \& Karmen, A. (1986) Activity concentration and mass concentration (monoclonal antibody immunoenzymometric method) compared for creatine kinase MB isoenzyme in serum. Clin. Chem. 32, 1956-1959.

14. Christenson, R. H., Clemmensen, P., Ohman, E. M., Toffaletti, J., Silverman, L. M., Grande, P. \& Wagner, G. S. (1990) Relative increase in creatine kinase MB isoenzyme during reperfusion after myocardial infarction is method dependent. Clin. Chem. 36, 1444-1449.

15. Panteghini, M. \& Pagani, F. (1988) Comparison of catalytic activity and mass concentration of serum creatine kinase $\mathrm{MB}$ isoenzyme in the detection of coronary reperfusion in acute myocardial infarction after therapeutic thrombolysis. J. Clin. Chem. Clin. Biochem. 26, 277-280.

Dr. Vibeke Schiøler

Department of Clinical Chemistry

Herlev University Hospital

DK-2730 Herlev 
